\title{
A CONCENTRIC HYDRODYNAMIC JOURNAL BEARING WITH THE BOUNDARY SLIPPAGE
}

\author{
YONGBIN ZHANG \\ College of Mechanical Engineering, Changzhou University, Changzhou, Jiangsu Province, China \\ e-mail: engmech1@sina.com
}

\begin{abstract}
The paper proposes a concentric hydrodynamic journal bearing constructed by the boundary slippage, which is opposed by conventional lubrication theory. Analysis for the carried load and friction coefficient of this bearing is presented. The optimum condition for the maximum load-carrying capacity of this bearing is examined. It is shown that the whole circumference of the bearing should be taken as the lubricated area, while on most of the stationary surface of the bearing there should be a hydrophobic coating covered so that the boundary slippage would occur on this surface, In this condition, the load-carrying capacity of the bearing is the highest but its friction coefficient is the lowest.
\end{abstract}

Keywords: hydrodynamics, boundary slippage, load, friction, bearing

\section{Introduction}

Conventional lubrication theory says that no hydrodynamic lubrication effect can be generated between two sliding parallel smooth plane surfaces (Pinkus, 1961). It also denies a concentric hydrodynamic journal bearing, where the lubricating film thickness is circumferentially constant. However, in practice, a concentric hydrodynamic journal bearing is very useful because of its high supporting precision, high lubricating film thickness, low viscous friction and low energy consumption.

The boundary slippage has been found to be capable of improving the performance of a hydrodynamic lubrication (Salant and Fortier, 2004; Zhang, 2008, 2010, 2013, 2014, 2015b; $\mathrm{Li}$ et al, 2014). It was found that hydrodynamic lubrication can be generated between two sliding parallel smooth plane surfaces because of the boundary slippage (Zhang, 2008). While, in conventional bearing configurations, the artificial introduction of the boundary slippage can increase the load-carrying capacity of the bearing but reduce its friction coefficient Zhang (2010, 2013, 2014, 2015b). In Zhang (2015b), the performance of a hydrodynamic journal bearing with an eccentricity was found to be able to be significantly improved by the boundary slippage. However, in that paper, a concentric hydrodynamic journal bearing was not addressed.

This paper proposes a concentric hydrodynamic journal bearing which is formed dependent on the boundary slippage. Analysis of this bearing is presented. It has been found that a significant load-carrying capacity can be generated depending on the design method applied. The optimum condition for the maximum load-carrying capacity of the bearing is also analyzed. In this optimum condition, the bearing also works with the lowest friction coefficient. The study shows a potential application value of such a bearing in practice.

\section{The bearing configuration}

Figure 1 shows the configuration of the studied bearing. The bearing is formed by a rotating shaft (with circumferential speed $u$ ) and a stationary sleeve. The two elements are concentric 
and the lubricating film thickness in the bearing is constant and equal to the bearing clearance $c(=R-r)$. The clearance of the bearing is filled with a fluid. The lubricated area of the bearing is divided into two subzones, i.e. "I" and "II" subzones which are, respectively, the inlet and outlet zones of the bearing. On the stationary (sleeve) surface in "I" subzone there is a hydrophobic coating covered to yield a low fluid film-contact surface interfacial shear strength $\left(\tau_{s a}\right)$ at this surface so that the boundary slippage would occur at this coated surface. The envelope angles of "I" and "II" subzones are respectively $\phi_{I}$ and $\phi_{I I}$. On the other surfaces of the bearing, the fluid film-contact surface interfacial shear strength is relatively high so that the boundary slippage is absent on these surfaces. The radii of the shaft and sleeve are $r$ and $R$, respectively. The carried load per unit contact width and attitude angle of the bearing are respectively $w$ and $\gamma$. The coordinate system used in the analysis is also shown in Fig. 1.

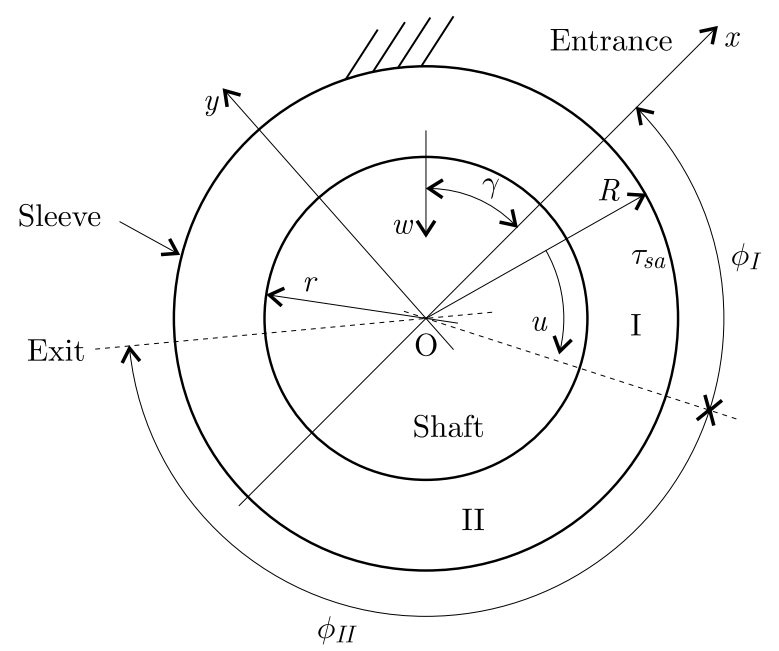

The bearing is filled with a fluid between the shaft and the sleeve. The fluid film slips at the sleeve surface in subzone "I" beacause of low fluid-sleeve interfacial shear strength $\tau_{s a}$. It does not slip at the other bearing surfaces because of relatively hight interfacial shear strength there

Fig. 1. Configuration of the proposed bearing

\section{Analysis}

The analysis carried out by Zhang (2015b) is also applicable to the present bearing. The analysis is based on the following assumptions:

(a) The lubricant film thickness is high enough so that the lubricant is continuum across the film thickness;

(b) The lubricant film is Newtonian within the film;

(c) The lubricant is isoviscous and imcompressible;

(d) Contact surface deformations are negligible;

(e) The side leakage in the bearing is negligible and the lubricant is in laminar flow;

(f) The operating condition is isothermal.

Accordingly to Zhang (2015b), the following dimensionless parameters are defined:

$$
W=\frac{w c^{2}}{u \eta r^{2}} \quad P=\frac{p c^{2}}{u \eta r} \quad Q_{v}=\frac{q_{v}}{u c} \quad \bar{F}_{x}=\frac{F_{x} c^{2}}{u \eta r^{2}} \quad \bar{F}_{y}=\frac{F_{y} c^{2}}{u \eta r^{2}}
$$




$$
\bar{F}_{f, h}=\frac{F_{f, h} c^{2}}{u \eta r^{2}} \quad \bar{F}_{f, s}=\frac{F_{f, s} c^{2}}{u \eta r^{2}} \quad \bar{\tau}=\frac{\tau c^{2}}{u \eta r} \quad k_{\tau}=\frac{\tau_{s a} c}{u \eta} \quad D U=\frac{\Delta u}{u}
$$

Here, $\eta$ is fluid viscosity, $p$ is film pressure, $q_{v}$ is volume flow rate in the bearing per unit contact width, $F_{x}$ and $F_{y}$ are respectively components of the carried load in the $x$ and $y$ coordinate directions, $F_{f, h}$ and $F_{f, s}$ are respectively friction forces per unit contact width acting on the sleeve and shaft surfaces, $\tau$ is shear stress, and $\Delta u$ is fluid film interfacial slipping velocity.

The pressure boundary conditions in the bearing are:

$$
\left.P\right|_{\phi=0}=\left.0 \quad P\right|_{\phi=\phi_{I}+\phi_{I I}}=0
$$

When the eccentricity ratio $\varepsilon$ is zero, a lot of the analytical results obtained by Zhang (2015b) are applicable to the present bearing. The following Sections demonstrate those results.

\section{1. "I" subzone}

The dimensionless Reynolds equation in "I" subzone is:

$$
\frac{d P_{\text {slip }}}{d \phi}=3-3 Q_{v, \text { slip }}-\frac{3 k_{\tau}}{2}
$$

Using the boundary condition in Eq. (3.1), integrating Eq. (3.2) gives dimensionless pressure in "I" subzone:

$$
P_{\text {slip }}=\left(3-3 Q_{v, \text { slip }}-\frac{3 k_{\tau}}{2}\right) \phi \quad \text { for } \quad 0 \leqslant \phi \leqslant \phi_{I}
$$

The dimensionless pressure on the boundary between "I" and "II" subzones is:

$$
P_{s l i p, \max }=\left(3-3 Q_{v, s l i p}-\frac{3 k_{\tau}}{2}\right) \phi_{I}
$$

\section{2. "II" subzone}

The dimensionless Reynolds equation in "II" subzone is:

$$
\frac{d P_{\text {slip }}}{d \phi}=6-12 Q_{v, \text { slip }}
$$

Using the boundary condition in Eq. (3.1), integrating Eq. (3.5) gives dimensionless pressure in "II" subzone:

$$
P_{\text {slip }}=\left(6-12 Q_{v, \text { slip }}\right)\left(\phi-\phi_{I}-\phi_{I I}\right) \quad \text { for } \quad \phi_{I} \leqslant \phi \leqslant \phi_{I}+\phi_{I I}
$$

According to Eq. (3.6), the dimensionless pressure on the boundary between "I" and "II" subzones is:

$$
P_{s l i p, \max }=\left(12 Q_{v, s l i p}-6\right) \phi_{I I}
$$

Equations (3.3) and (3.6) show that the pressure is respectively linearly distributed in "I" and "II" subzones in the present bearing. Figure 2 schematically shows the pressure distribution in the present bearing. 


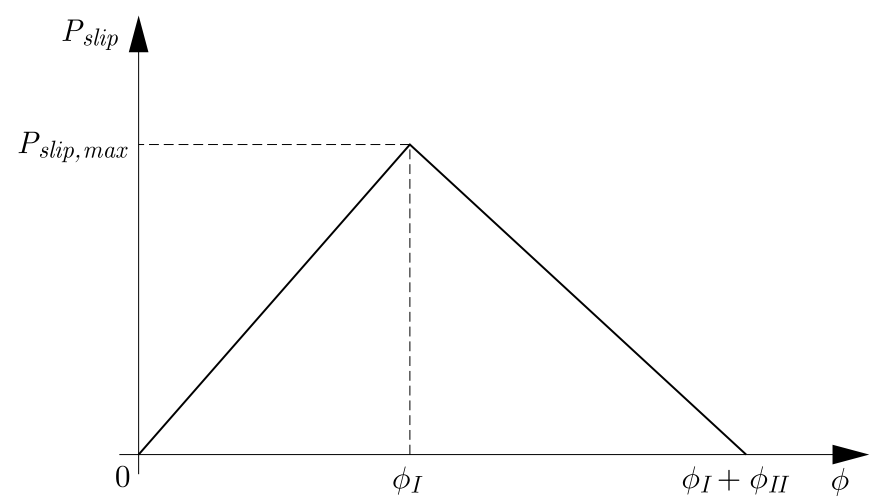

Fig. 2. Illustration of the pressure distribution in the proposed bearing

\subsection{Volume flow rate and condition for the bearing}

Define $\psi_{\phi}=\phi_{I I} / \phi_{I}$, solving coupled equations (3.4) and (3.7) gives:

$$
Q_{v, s l i p}=\frac{1+2 \psi_{\phi}-\frac{1}{2} k_{\tau}}{1+4 \psi_{\phi}}
$$

and

$$
P_{\text {slip }, \max }=\bar{P}_{\text {slip }, \max }\left(\phi_{I}+\phi_{I I}\right)
$$

where $\bar{P}_{\text {slip, } \max }=6 \psi_{\phi}\left(1-k_{\tau}\right) /\left[\left(1+\psi_{\phi}\right)\left(1+4 \psi_{\phi}\right)\right]$.

From $Q_{v, \text { slip }}>0$, it is obtained that $k_{\tau}<2+4 \psi_{\phi}$. From $P_{\text {slip, max }}>0$, it is obtained that $k_{\tau}<1$. Therefore, $k_{\tau}<1$ is the condition for the present bearing.

It is noted from Eq. (3.9) that for given values of $k_{\tau}$ and $\phi_{I}+\phi_{I I}$, when $\psi_{\phi}=1 / 2, P_{\text {slip, } \max }$ reaches the maximum, and its maximum value is $2\left(1-k_{\tau}\right)\left(\phi_{I}+\phi_{I I}\right) / 3$.

\subsection{Carried load and attitude angle of the bearing}

The dimensionless hydrodynamic force component in the $x$ axis direction acting on the shaft per unit contact width is:

$$
\bar{F}_{x, \text { slip }}=-\int_{0}^{\phi_{I}+\phi_{I I}} P_{\text {slip }} \cos \phi d \phi=\bar{P}_{\text {slip, } \max } f_{1}\left(\psi_{\phi}, \phi_{t o t}\right)
$$

where $\phi_{t o t}=\phi_{I}+\phi_{I I}$ and (also Zhang (2015a))

$$
\begin{gathered}
f_{1}\left(\psi_{\phi}, \phi_{t o t}\right)=\left(1+\frac{1}{\psi_{\phi}}\right)\left[\frac{\psi_{\phi} \phi_{t o t}}{1+\psi_{\phi}} \sin \left(\frac{\phi_{t o t}}{1+\psi_{\phi}}\right)+\cos \phi_{t o t}-\cos \left(\frac{\phi_{t o t}}{1+\psi_{\phi}}\right)\right] \\
-\left(1+\psi_{\phi}\right)\left[\frac{\phi_{t o t} \sin \left(\frac{\phi_{t o t}}{1+\psi_{\phi}}\right)}{1+\psi_{\phi}}+\cos \left(\frac{\phi_{t o t}}{1+\psi_{\phi}}\right)-1\right]
\end{gathered}
$$

The dimensionless hydrodynamic force component in the $y$ axis direction acting on the shaft per unit contact width is:

$$
\bar{F}_{y, s l i p}=\int_{0}^{\phi_{I}+\phi_{I I}} P_{\text {slip }} \sin \phi d \phi=\bar{P}_{\text {slip, max }} f_{2}\left(\psi_{\phi}, \phi_{\text {tot }}\right)
$$


where (also Zhang (2015a))

$$
\begin{gathered}
f_{2}\left(\psi_{\phi}, \phi_{t o t}\right)=\left(1+\frac{1}{\psi_{\phi}}\right)\left[\frac{\psi_{\phi} \phi_{t o t}}{1+\psi_{\phi}} \cos \left(\frac{\phi_{t o t}}{1+\psi_{\phi}}\right)-\sin \phi_{t o t}+\phi_{t o t} \cos \phi_{t o t}+\sin \left(\frac{\phi_{t o t}}{1+\psi_{\phi}}\right)\right] \\
+\left(1+\psi_{\phi}\right)\left[\sin \left(\frac{\phi_{t o t}}{1+\psi_{\phi}}\right)-\frac{\phi_{t o t}}{1+\psi_{\phi}} \cos \left(\frac{\phi_{t o t}}{1+\psi_{\phi}}\right)\right]
\end{gathered}
$$

The dimensionless load per unit contact width carried by the bearing is:

$$
W_{\text {slip }}=\sqrt{\bar{F}_{x, \text { slip }}^{2}+\bar{F}_{y, \text { slip }}^{2}}=\bar{P}_{\text {slip }, \max } f_{w}\left(\psi_{\phi}, \phi_{\text {tot }}\right)
$$

where $f_{w}\left(\psi_{\phi}, \phi_{t o t}\right)=\sqrt{f_{1}^{2}\left(\psi_{\phi}, \phi_{t o t}\right)+f_{2}^{2}\left(\psi_{\phi}, \phi_{t o t}\right)}$ (Zhang, 2015a).

Figure 3 plots the values of $f_{w}$ against $\phi_{\text {tot }}$ for the given values of $\psi_{\phi}$. It is shown that for a given $\psi_{\phi}$, the value of $f_{w}$ reaches the maximum when $\phi_{t o t}=2 \pi$. This means that for the maximum load-carrying capacity of the bearing, $\phi_{\text {tot }}$ should be taken as $2 \pi$.

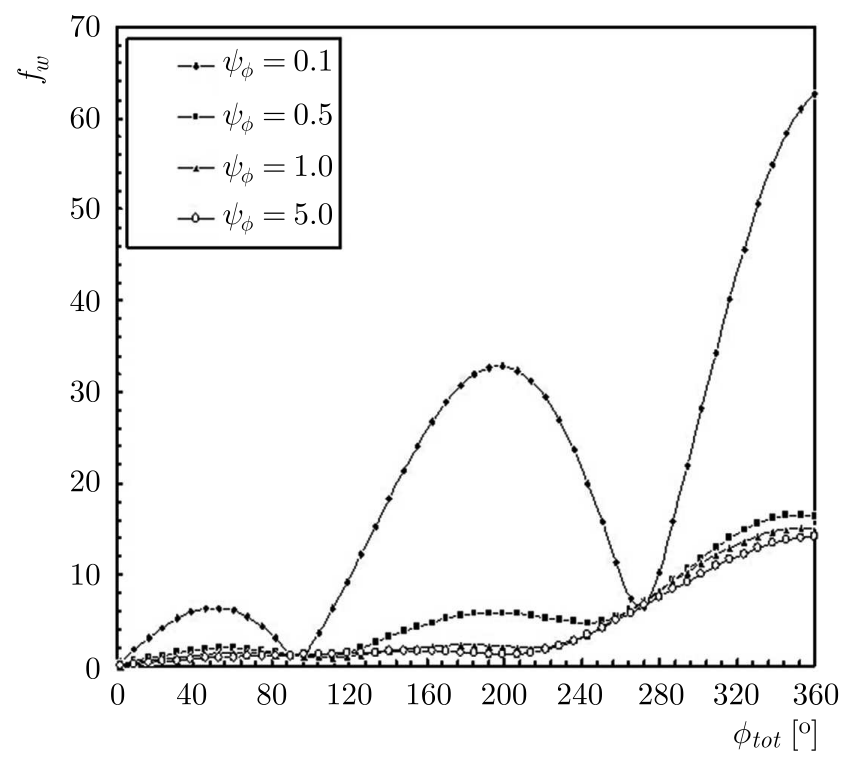

Fig. 3. Plots of $f_{w}$ against $\phi_{t o t}$ for given $\psi_{\phi}$ values (Zhang, 2015a)

When $\phi_{t o t}=2 \pi$, the dimensionless load is:

$$
W_{\text {slip }}=\left(1-k_{\tau}\right) G\left(\psi_{\phi}\right)
$$

where $G\left(\psi_{\phi}\right)=6 \psi_{\phi} f_{w}\left(\psi_{\phi}, 2 \pi\right) /\left[\left(1+\psi_{\phi}\right)\left(1+4 \psi_{\phi}\right)\right]$.

The attitude angle of the bearing is:

$$
\gamma=\arctan \left[\frac{f_{2}\left(\psi_{\phi}, \phi_{t o t}\right)}{f_{1}\left(\psi_{\phi}, \phi_{t o t}\right)}\right]
$$

\subsection{Friction coefficient and interfacial slipping velocity}

The dimensionless shear stress on the shaft surface is:

$$
\bar{\tau}_{s, \text { slip }}= \begin{cases}\frac{c}{r}\left(3-\frac{k_{\tau}}{2}-3 Q_{v, \text { slip }}\right) & \text { for } \quad 0 \leqslant \phi \leqslant \phi_{I} \\ \frac{c}{r}\left(4-6 Q_{v, \text { slip }}\right) & \text { for } \quad \phi_{I}<\phi \leqslant \phi_{I}+\phi_{I I}\end{cases}
$$


The dimensionless shear stress on the sleeve surface is:

$$
\bar{\tau}_{h, \text { slip }}= \begin{cases}k_{\tau} \frac{c}{r} & \text { for } \quad 0 \leqslant \phi \leqslant \phi_{I} \\ \frac{c}{r}\left(6 Q_{v, \text { slip }}-2\right) & \text { for } \quad \phi_{I}<\phi \leqslant \phi_{I}+\phi_{I I}\end{cases}
$$

The dimensionless friction force on the shaft surface per unit contact width is:

$$
\begin{gathered}
\bar{F}_{f, s, \text { slip }}=\int_{0}^{\phi_{I}+\phi_{I I}} \bar{\tau}_{s, \text { slip }} d \phi=\int_{0}^{\phi_{I}} \bar{\tau}_{s, \text { slip }} d \phi+\int_{\phi_{I}}^{\phi_{I}+\phi_{I I}} \bar{\tau}_{s, \text { slip }} d \phi \\
=\frac{c}{r}\left(3-\frac{k_{\tau}}{2}-3 Q_{v, \text { slip }}\right) \phi_{I}+\frac{c}{r}\left(4-6 Q_{v, \text { slip }}\right) \phi_{I I}
\end{gathered}
$$

The dimensionless friction force on the sleeve surface per unit contact width is:

$$
\begin{gathered}
\bar{F}_{f, h, s l i p}=\int_{0}^{\phi_{I}+\phi_{I I}} \bar{\tau}_{h, \text { slip }} d \phi=\int_{0}^{\phi_{I}} \bar{\tau}_{h, \text { slip }} d \phi+\int_{\phi_{I}}^{\phi_{I}+\phi_{I I}} \bar{\tau}_{h, s l i p} d \phi \\
=k_{\tau} \phi_{I} \frac{c}{r}+\frac{c}{r}\left(6 Q_{v, \text { slip }}-2\right) \phi_{I I}
\end{gathered}
$$

The friction coefficients on the sleeve and shaft surfaces are respectively:

$$
f_{h, \text { slip }}=\frac{\bar{F}_{f, h, \text { slip }}}{W_{\text {slip }}} \quad f_{s, \text { slip }}=\frac{\bar{F}_{f, s, \text { slip }}}{W_{\text {slip }}}
$$

The dimensionless slipping velocity of the fluid film at the sleeve surface is:

$$
D U= \begin{cases}\frac{3 Q_{v, \text { slip }}}{2}-\frac{1}{2}-\frac{k_{\tau}}{4} & \text { for } \quad 0 \leqslant \phi \leqslant \phi_{I} \\ 0 & \text { for } \quad \phi_{I}<\phi \leqslant \phi_{I}+\phi_{I I}\end{cases}
$$

where $D U$ should be positive for $0 \leqslant \phi \leqslant \phi_{I}$.

\section{Results and discussion}

Figure 4a plots the values of $G$ against $\psi_{\phi}$ when $\phi_{t o t}=2 \pi$. It is shown that $G$ significantly increases with the reduction of $\psi_{\phi}$ when $\psi_{\phi} \geqslant 0.1$. While, for $\psi_{\phi}<0.01, G$ is weakly influenced by $\psi_{\phi}$. According to Eq. (3.15), it means that for a given $k_{\tau}$ the load-carrying capacity of the bearing increases with the reduction of $\psi_{\phi}$, especially when $\psi_{\phi} \geqslant 0.1$, while too low values of $\psi_{\phi}$ have no benefits in increasing the load-carrying capacity. As the optimum value of $\psi_{\phi}$ for the maximum value of $P_{\text {slip, max }}$ is 0.5 , in the engineering design, the value of $\psi_{\phi}$ may be recommended to be chosen between 0.1 and 0.5 .

Figure $4 \mathrm{~b}$ plots values of $\gamma$ against $\psi_{\phi}$ when $\phi_{\text {tot }}=2 \pi$. The minimum value of $\gamma$ is about $57^{\circ}$, and it occurs when $\psi_{\phi}$ is around 1.0. For $\psi_{\phi}<0.1$ or $\psi_{\phi}>20, \gamma$ approaches $90^{\circ}$.

Figures 5a and 5b plot respectively values of $f_{s, s l i p} r / c$ and $f_{h, s l i p} r / c$ against $\psi_{\phi}$ for different $k_{\tau}$ when $\phi_{t o t}=2 \pi$. It is shown that for given values of $k_{\tau}$ and $c / r$, the friction coefficients $f_{s, s l i p}$ and $f_{h, s l i p}$ both are linearly reduced with the reduction of $\psi_{\phi}$. This indicates that a relatively low value of $\psi_{\phi}$ has also benefit of giving a low friction coefficient to the bearing. The reduction of $k_{\tau}$ is shown to significantly reduce the friction coefficient, especially when $\psi_{\phi}$ is high. 
(a)

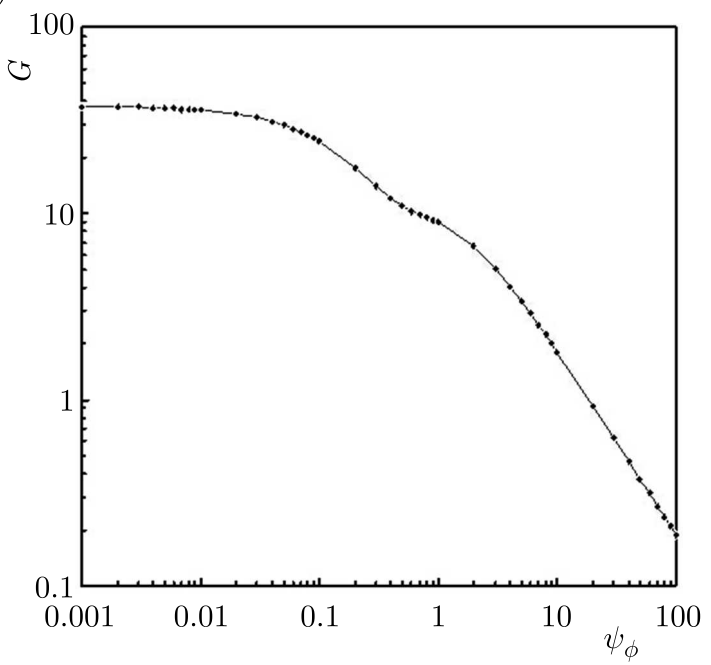

(b)

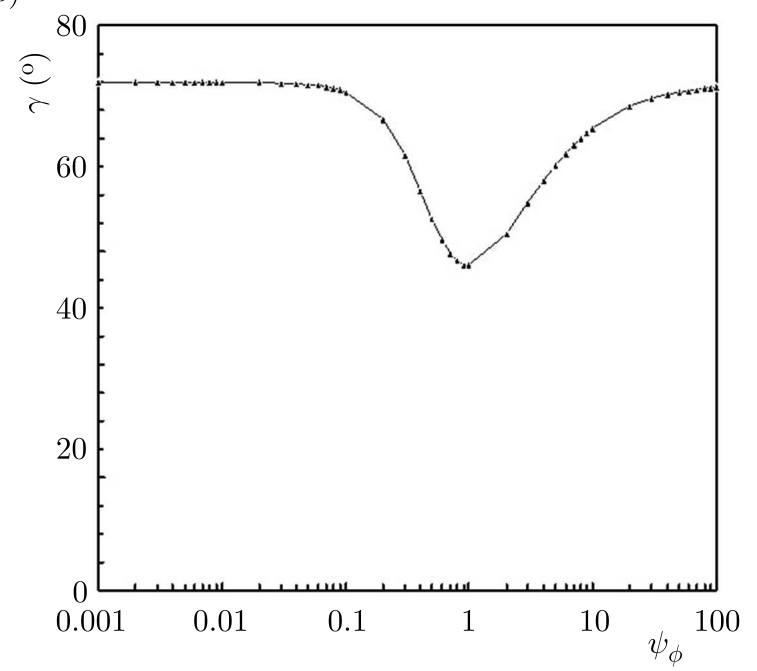

Fig. 4. Plots of (a) $G$, (b) $\gamma$ against $\psi_{\phi}$ when $\phi_{t o t}=2 \pi$

(a)

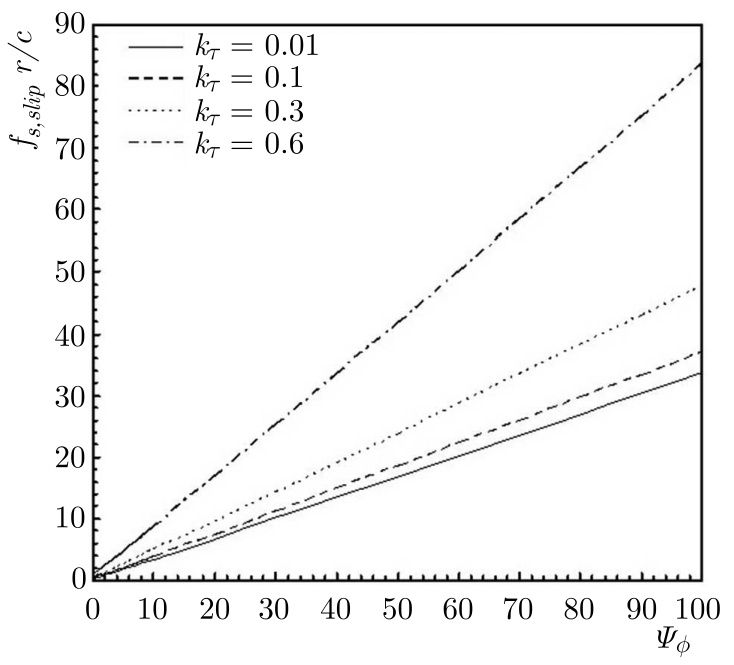

(b)

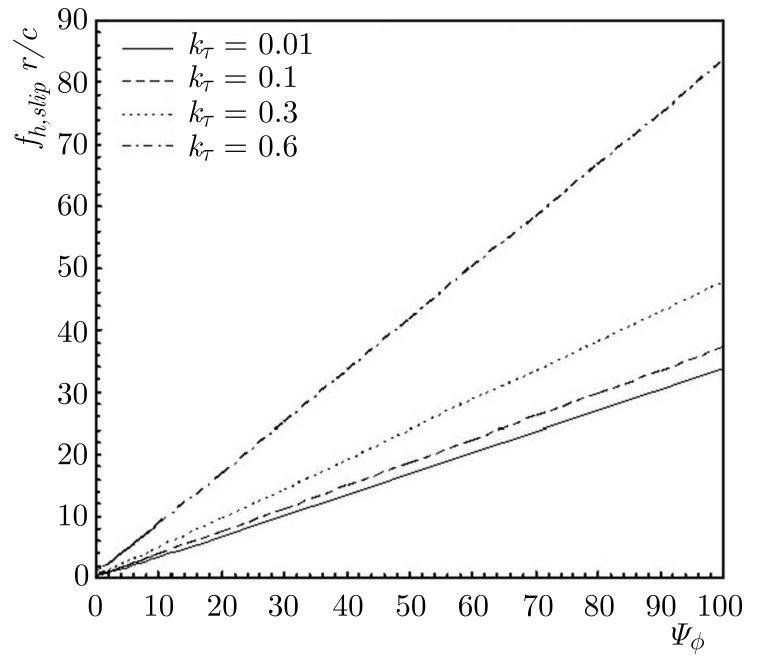

Fig. 5. Plots of $f_{s, s l i p} r / c$ and $f_{h, s l i p} r / c$ against $\psi_{\phi}$ for different $k_{\tau}$ when $\phi_{t o t}=2 \pi$

\section{Conclusions}

This paper proposes a concentric hydrodynamic journal bearing which is formed dependent on the boundary slippage. The configuration of the bearing is presented. The lubricated area of the bearing is divided into two subzones, which may respectively be the inlet and outlet zones. In the inlet zone, on the stationary surface a hydrophobic coating is covered to yield a low fluid film-contact surface interfacial shear strength so that the boundary slippage could occur on this surface. On the other bearing surfaces, the boundary slippage is absent because of relatively high interfacial shear strengths on these surfaces.

Analysis for the load-carrying capacity and friction coefficient of the bearing is presented. Typical calculations have been carried out. It has been found that the optimum value of the ratio of the circumferential length of the outlet zone to that of the inlet zone, i.e. the optimum value of $\psi_{\phi}$ is 0.5 for the maximum hydrodynamic pressure building-up. However for this value $\psi_{\phi}$, the load-carrying capacity of the bearing is still not the maximum. The whole circumference of the bearing should be taken as the lubricated area for achieving a high load-carrying capacity. 
In this condition, the carried load of the bearing is found to be increased with the reduction of $\psi_{\phi}$, especially when $\psi_{\phi} \geqslant 0.1$. Nevertheless, for $\psi_{\phi} \leqslant 0.01$, the load-carrying capacity of the bearing is weakly influenced by variation of $\psi_{\phi}$. It is recommended that in engineering design the value of $\psi_{\phi}$ should be chosen between 0.1 and 0.5 . A low value of $\psi_{\phi}$ also has the benefit of giving a low friction coefficient to the bearing.

\section{References}

1. Pinkus O., Sternlicht B., 1961, Theory of hydrodynamic lubrication, McGraw-Hill, New York

2. Salant R.F., Fortier A.E., 2004, Numerical analysis of a slider bearing with a heterogeneous slip/no-slip surface, Tribology Transactions, 47, 328-334

3. Zhang Y.B., 2008, Boundary slippage for generating hydrodynamic load-carrying capacity, Applied Mathematics and Mechanics, 29, 1155-1164

4. ZHANG Y.B., 2010, Boundary slippage for improving the load and friction performance of a step bearing, Transactions of the Canadian Society for Mechanical Engineering, 34, 373-387

5. Zhang Y.B., 2013, A tilted pad thrust slider bearing improved by boundary slippage, Meccanica, 48, 769-781

6. ZHANG Y.B., 2014, Hydrodynamic lubrication in line contacts improved by the boundary slippage, Meccanica, 49, 503-519

7. ZhANG Y.B., 2015a, A concentric micro/nano journal bearing constructed by physical adsorption, Journal of the Balkan Tribological Association, 21, 937-951

8. ZHANG Y.B., 2015b, An improved hydrodynamic journal bearing with the boundary slippage, Meccanica, 50, 25-38

9. Li G., Zhang Y.B., Jiang X.D., 2014, A study on the performance of $s$ hydrodynamic step bearing by controlling its boundary slippage in the outlet zone, Journal of Changzhou University, Natural Sciences Edition, 26, 33 